\title{
THE INFLUENCE OF GRAIN BOUNDARY ELEMENTS ON PROPERTIES AND MICROSTRUCTURES OF P/M NICKEL BASE SUPERALLOYS
}

\author{
E. S. Huron, K. R. Bain, D. P. Mourer ${ }^{1}$, J. J. Schirra, P. L. Reynolds, and E. E. Montero², \\ ${ }^{1}$ GE Aircraft Engines, Cincinnati, OH. \\ ${ }^{2}$ Pratt \& Whitney, East Hartford, CT.
}

Keywords: Grain boundary, grain boundary element, powder metallurgy, Boron, Carbon, Hafnium, Magnesium, Zirconium, Tantalum

\begin{abstract}
A study was conducted on the influence of grain boundary elements (GBE) on several $\mathrm{P} / \mathrm{M}$ nickel base superalloys. Evaluations included microstructural response to chemistry and processing, tensile, creep, rupture, and fatigue crack growth testing (FCG). Boron was found to be the most influential element in the study, with increased boron leading to increased grain boundary triple point void formation and tendency for Thermally Induced Porosity (TIP). Boron reduced creep life but increased both rupture life and rupture ductility and slightly reduced hold time crack growth rates. Carbon also influenced microstructure by slightly refining grain size, and slightly reduced creep and hold time crack growth rates. Hafnium had slight impacts on yield strength and hold time crack growth rates. Magnesium overall had negligible effects. Tantalum had several significant effects, improving creep life and reducing crack growth rates. Overall, the results confirm that careful control and optimization of grain boundary chemistries and grain boundary microstructure are necessary to achieve maximum performance of $\mathrm{P} / \mathrm{M}$ superalloys.
\end{abstract}

\section{Introduction}

In the mid-1990's, NASA began a program aimed at developing an engine for the High Speed Civil Transport (HSCT). This program included a materials-targeted effort called the Enabling Propulsion Materials (EPM) program and resulted in a joint contract effort between GE Aircraft Engines, Pratt \& Whitney, and NASA Glenn Research Center (formerly the NASA Lewis Center). The goal of the disk portion of the EPM effort was to develop a new turbine disk material for the HSCT mission; this mission can be generalized as long dwell fatigue and creep/rupture loading cycles at temperatures above 704C.

The project team reviewed prior published and internal superalloy data and experience and immediately realized that successfully meeting the project goals would require alloys beyond the thencurrent commercial alloys (such as IN100 and Rene' 88DT), with improvements required in both bulk element composition and in grain boundary chemistry and structure. The team began two parallel studies to begin the effort, one based on microstructural modification of several promising superalloys, and one based on grain boundary optimization. This paper describes the grain boundary element study while the microstructural study is described in a parallel paper in this volume ${ }^{1}$.

\section{Procedure}

The study used several promising matrix alloys as starting points. All of the alloys were high volume fraction (over 50\%) gamma- prime ( $\left.\gamma^{\prime}\right)$ nickel-base superalloys processed via powder metallurgy $(\mathrm{P} / \mathrm{M})$. Two matrices were studied. In the first, grain boundary element levels were varied using a nominal composition based on $18 \mathrm{Co}-12 \mathrm{Cr}-3.9 \mathrm{Mo}-\mathrm{xNb}-4 \mathrm{Al}-4 \mathrm{Ti}$ (all compositions in this paper are in weight percent). The core experiment used $\mathrm{KM} 4^{2}$, a GE-developed alloy containing $2 \mathrm{w} / \mathrm{o} \mathrm{Nb}$ and an $\mathrm{Al} / \mathrm{Ti}$ ratio of 1:1; one minor element level was also evaluated for alloy $456^{3}$, a Pratt \& Whitney-developed alloy with an addition of $\mathrm{W}$ and a higher $\mathrm{Al} / \mathrm{Ti}$ ratio, with the $\mathrm{Nb}$ level of 1 weight percent; and one minor element level was evaluated for $\mathrm{CH}_{98}^{4}$, a GEdeveloped alloy with an addition of 4 w/o Ta. The base compositions were slightly modified in the second matrix; in particular they contained no $\mathrm{Nb}$. The second matrix also included a single chemistry with a lower $\mathrm{Co} / \mathrm{Cr}$ ratio and a lower $\mathrm{Al} / \mathrm{Ti}$ ratio. The 456 and $\mathrm{CH} 98$ alloys had been under development in prior programs and were added for comparison.

With the above as core compositions, the grain boundary elements were systematically varied in two statistically designed experiments, Matrix 1 and Matrix 2. The experimental matrix designs are shown in Tables I and II. In the first matrix, high and low levels of $\mathrm{C}, \mathrm{B}, \mathrm{Hf}$, and $\mathrm{Mg}$ were evaluated. The aim levels were varied between 0.01 and 0.04 boron, 0.02 and 0.06 carbon, zero and 0.30 hafnium, and zero and 0.005 magnesium.

Table I: Matrix 1 Experimental Design

\begin{tabular}{|l|l|l|l|l|}
\hline Alloy & \multicolumn{1}{|c|}{$\mathrm{B}$} & \multicolumn{1}{c|}{$\mathrm{C}$} & \multicolumn{1}{c|}{$\mathrm{Hf}$} & \multicolumn{1}{c|}{$\mathrm{Mg}$} \\
\hline & \multicolumn{1}{|c|}{ Base 12Cr-18Co-4Mo-2Nb-4Al-4Ti-0.030Zr } \\
\hline KM4-GB1 & $\mathrm{Lo} / 0.01$ & $\mathrm{Lo} / 0.02$ & $\mathrm{Lo} / 0.0$ & $\mathrm{Lo} / 0.0$ \\
\hline KM4-GB2 & $\mathrm{Hi} / 0.04$ & $\mathrm{Lo} / 0.02$ & $\mathrm{Lo} / 0.0$ & $\mathrm{Hi} / 0.005$ \\
\hline KM4-GB3 & $\mathrm{Lo} / 0.01$ & $\mathrm{Hi} / 0.06$ & $\mathrm{Lo} / 0.0$ & $\mathrm{Hi} / 0.005$ \\
\hline KM4-GB4 & $\mathrm{Hi} / 0.04$ & $\mathrm{Hi} / 0.06$ & $\mathrm{Lo} / 0.0$ & $\mathrm{Lo} / 0.0$ \\
\hline KM4-GB5 & $\mathrm{Lo} / 0.01$ & $\mathrm{Lo} / 0.02$ & $\mathrm{Hi} / 0.3$ & $\mathrm{Hi} / 0.005$ \\
\hline KM4-GB6 & $\mathrm{Hi} / 0.04$ & $\mathrm{Lo} / 0.02$ & $\mathrm{Hi} / 0.3$ & $\mathrm{Lo} / 0.0$ \\
\hline KM4-GB7 & $\mathrm{Lo} / 0.01$ & $\mathrm{Hi} / 0.06$ & $\mathrm{Hi} / 0.3$ & $\mathrm{Lo} / 0.0$ \\
\hline KM4-GB8 & $\mathrm{Hi} / 0.04$ & $\mathrm{Hi} / 0.06$ & $\mathrm{Hi} / 0.3$ & $\mathrm{Hi} / 0.005$ \\
\hline $\begin{array}{l}\text { KM4-GB- } \\
\text { CP }\end{array}$ & 0.025 & 0.04 & 0.150 & 0.0025 \\
\hline $\begin{array}{l}\text { KM4-GB- } \\
\text { Base }\end{array}$ & 0.03 & 0.03 & 0 & 0 \\
\hline KM4-Zr & 0.03 & 0.03 & $0.07 \mathrm{Zr}(\mathrm{no} \mathrm{Hf} \mathrm{or} \mathrm{Mg)}$ \\
\hline 456-GB8 & 456 base $+0.05 \mathrm{~B} / 0.07 \mathrm{C} / 0.3 \mathrm{Hf} / 0.005 \mathrm{Mg}$ \\
\hline CH98 & $\mathrm{CH} 98$ base $+0.03 \mathrm{~B} / 0.03 \mathrm{C} / 0 \mathrm{Hf} / 0 \mathrm{Mg}$ \\
\hline
\end{tabular}

In the second experiment, carbon, boron, $\mathrm{Ta}$, and $\mathrm{Mg}$ were varied. The average carbon and boron levels in the second experiment were higher than in the first experiment; the upper $\mathrm{Mg}$ level in the second experiment was higher to screen for potential missed effects from the low level in the first experiment. 
For both experiments, material was processed using standard $\mathrm{P} / \mathrm{M}$ techniques, using gas atomized powder. The powder for Matrix 1 was produced using a laboratory scale atomizer at GE Global Research Center (GE-GRC) in Schenectady, NY. For each composition, three vacuum induction melted (VIM) ingots for each composition were prepared. Multiple powder lots were produced and powder was screened and then blended to yield a single blend of the desired chemistry. For Matrix 2, powder lots were produced using a pilot-scale atomizer at Homogeneous Metals, Clayville, NY. Only a single heat was required to produce sufficient powder. Chemistries obtained generally matched aims. These results are shown in Tables III and IV.

Table II: Matrix 2 Experimental Design

\begin{tabular}{|l|l|l|l|l|}
\hline Alloy & \multicolumn{1}{|c|}{$\mathrm{B}$} & \multicolumn{1}{c|}{$\mathrm{C}$} & \multicolumn{1}{c|}{$\mathrm{Ta}$} & \multicolumn{1}{c|}{$\mathrm{Mg}$} \\
\hline & \multicolumn{3}{|l|}{ Base 12.1Cr-18.9Co-4Mo-4.2Al-4Ti-0.030Zr } \\
\hline GBE2-1 & $\mathrm{Lo} / 0.02$ & $\mathrm{Lo} / 0.03$ & $\mathrm{Lo} / 0.8$ & $\mathrm{Lo} / 0.0$ \\
\hline GBE2-2 & $\mathrm{Lo} / 0.02$ & $\mathrm{Hi} / 0.055$ & $\mathrm{Lo} / 0.8$ & $\mathrm{Hi} / 0.0075$ \\
\hline GBE2-3 & $\mathrm{Hi} / 0.04$ & $\mathrm{Lo} / 0.03$ & $\mathrm{Lo} / 0.8$ & $\mathrm{Hi} / 0.0075$ \\
\hline GBE2-4 & $\mathrm{Hi} / 0.04$ & $\mathrm{Hi} / 0.055$ & $\mathrm{Lo} / 0.8$ & $\mathrm{Lo} / 0.0$ \\
\hline GBE2-5 & $\mathrm{Lo} / 0.02$ & $\mathrm{Lo} / 0.03$ & $\mathrm{Hi} / 2.4$ & $\mathrm{Hi} / 0.0075$ \\
\hline GBE2-6 & $\mathrm{Lo} / 0.02$ & $\mathrm{Hi} / 0.055$ & $\mathrm{Hi} / 2.4$ & $\mathrm{Lo} / 0.0$ \\
\hline GBE2-7 & $\mathrm{Hi} / 0.04$ & $\mathrm{Lo} / 0.03$ & $\mathrm{Hi} / 2.4$ & $\mathrm{Lo} / 0.0$ \\
\hline GBE2-8 & $\mathrm{Hi} / 0.04$ & $\mathrm{Hi} / 0.055$ & $\mathrm{Hi} / 2.4$ & $\mathrm{Hi} / 0.0075$ \\
\hline GBE2-9CP & 0.030 & 0.043 & 1.6 & 0.0038 \\
\hline GBE2-10 & $15 \mathrm{Cr}-17 \mathrm{Co}-5 \mathrm{Mo}-2.5 \mathrm{Al}-4.7 \mathrm{Ti}$ \\
& $+0.03 \mathrm{~B} / 0.06 \mathrm{C} / 3.07 \mathrm{Ta} / 0.0 \mathrm{Mg}$ \\
\hline
\end{tabular}

Powder for both matrices was consolidated by Wyman-Gordon in Houston, TX, using a pilot-scale extrusion press. Hot extrusion was done at 1093C. Mults were cut from each extrusion and isothermally forged. Matrix 1 mults were forged at WymanGordon and Matrix 2 mults were forged at Pratt \& Whitney in West Palm Beach, FL. All forgings were produced at 1066C. The pancake sizes were nominally $2.8 \mathrm{~cm}$ thick $\mathrm{x} 16.5 \mathrm{~cm}$ diameter. Two forgings were produced from each extrusion. Coupons from forgings and extrusions were used for various heat treatment response, solvus determination, and Thermally Induced Porosity (TIP) experiments, as described in subsequent sections. To produce material for the full property evaluation matrix for each composition, thermocoupled pancake experiments were performed to develop cooling procedures to achieve desired cooling rates. For both matrices, pancakes were removed from the furnace and cooled in still air to produce cooling rates in the pancake cores ranging from 77 to 103 degrees $\mathrm{C}$ per minute. The Matrix 1 pancakes were given supersolvus heat treatments of $1191 \mathrm{C} / 4 \mathrm{hrs}$ except for $\mathrm{CH} 98$ which was heat treated at $1218 \mathrm{C}$. For matrix 2, the procedure was similar except the supersolvus temperatures were adjusted slightly: 1191C for alloys \#1-4 and $\# 10$ and $1204 \mathrm{C}$ for alloys \#5-9. All of the pancakes were stabilized and aged at $843 \mathrm{C} / 4 \mathrm{hrs} /$ aircool $+760 \mathrm{C} / 4 \mathrm{hrs} /$ aircool.

Heat treatments were conducted using the forged material for Matrix 1, with some limited evaluations on Matrix 2. A TIP study was done with initial screening at 1093C and 1204C for 3 hours followed by a more detailed study at $14 \mathrm{C}$ intervals between $1135 \mathrm{C}$ and $1246 \mathrm{C}$. The experiments below $1204 \mathrm{C}$ were done in an air atmosphere while experiments at higher temperatures were done in argon. The evaluations included grain size vs. temperature, $\gamma$ ' solvus observation, incipient melting, and TIP. Grain size was measured using standard intercept methods and converted to an ASTM grain size number. Incipient melting was determined as the temperature at which, via 500X optical metallography, significant grain boundary decoration or films were seen or the formation of significant triple point pores was evident. For TIP, two measurements were made. One was a subjective rating scale of 1 (very little porosity) to 3 (obvious triple point porosity). The second was a well-established GEGRC test using density (measured by volumetric displacement) of cubes before and after exposure at $1204 \mathrm{C} / 4 \mathrm{hrs} /$ argon atmosphere. The density change is a measure of the degree of TIP.

The pancake forgings that were heat treated to provide material for specimens for various mechanical property testing were sectioned into blanks and machined into test bars for 649C tensile and stress rupture tests, $649 \mathrm{C} / 793 \mathrm{MPa}$ creep tests and $704 \mathrm{C}$ dwell fatigue crack growth tests.

\section{Results}

The results showed significant impact of the grain boundary elements on response to processing and heat treatment, microstructure, and mechanical properties. The results were analyzed with both simple comparison parameters to observe trends as well as with more detailed statistical analysis to determine which factors were "real" effects beyond normal variation. However, because a study of this nature involves a high degree of inherent, multiple-cause scatter, observed trends that are not conclusively statistically significant will still be discussed.

Cooling $\gamma^{\prime}$ sizes were observed to be uniform within each matrix, showing relatively consistent heat treat practice with each lot, but different between Matrix 1 and Matrix 2. The $\gamma$ ' sizes were generally 0.35 to 0.50 microns in Matrix 1 and 0.25 to 0.4 microns in Matrix 2. The differences in $\gamma^{\prime}$ sizes were larger than expected and probably reflect differences in the detailed cooling path due to furnace removal, placement during cooling, and laboratory environment. Figure 1 shows a typical $\gamma^{\prime}$ structure.

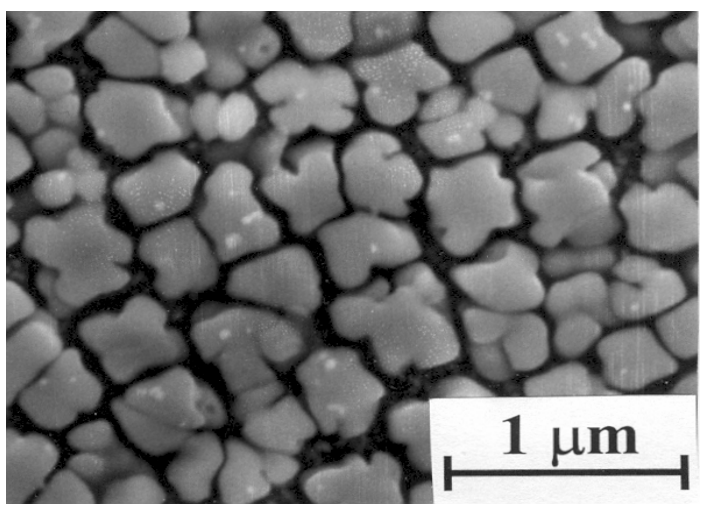

Figure 1: Typical $\gamma^{\prime}$ morphology from supersolvus solution plus air cool heat treatment. Alloy shown is KM4-GB8.

The initial microstructural observations for Matrix 1 were that, in general, the heat treated pancake microstructures had uniform, supersolvus grain size, relatively consistent across all compositions. Boron had a noticeable effect on grain boundary structure, with increased boron tending to promote grain boundary filming and triple point voiding. Hafnium led to grain boundary eutectic and $\mathrm{HfC}$ formation (Figure 2). 
Table III: Grain Boundary Element Matrix 1 Compositions (actual chemistries in w/o)

\begin{tabular}{|l|l|l|l|l|l|l|l|l|l|l|l|l|l|l|l|}
\hline Alloy & $\mathrm{B}$ & $\mathrm{C}$ & $\mathrm{S}$ & $\mathrm{Hf}$ & $\mathrm{Mg}$ & $\mathrm{Zr}$ & $\mathrm{Al}$ & $\mathrm{Ti}$ & $\mathrm{Ta}$ & $\mathrm{Nb}$ & $\mathrm{Cr}$ & $\mathrm{Co}$ & $\mathrm{Mo}$ & $\mathrm{W}$ & $\mathrm{Ni}$ \\
\hline $\begin{array}{l}\text { KM4- } \\
\text { Base }\end{array}$ & 0.025 & 0.0485 & 0.005 & 0 & 0.0019 & 0.0265 & 3.65 & 4.41 & 0 & 1.88 & 12.38 & 17.58 & 3.90 & 0 & bal \\
\hline $\begin{array}{l}\text { KM4- } \\
\text { GB1 }\end{array}$ & 0.0089 & 0.0240 & 0.001 & 0 & 0.0021 & 0.0270 & 3.67 & 4.40 & 0 & 1.96 & 12.58 & 16.24 & 4.03 & 0 & bal \\
\hline $\begin{array}{l}\text { KM4- } \\
\text { GB2 }\end{array}$ & 0.0230 & 0.0350 & 0.001 & 0 & 0.0122 & 0.0260 & 3.46 & 4.26 & 0 & 1.88 & 12.44 & 17.66 & 3.87 & 0.11 & bal \\
\hline $\begin{array}{l}\text { KM4- } \\
\text { GB3 }\end{array}$ & 0.0094 & 0.0624 & 0.001 & 0 & 0.0009 & 0.0260 & 3.68 & 4.26 & 0 & 1.94 & 12.49 & 17.58 & 3.97 & 0.02 & bal \\
\hline $\begin{array}{l}\text { KM4- } \\
\text { GB4 }\end{array}$ & 0.0314 & 0.0610 & 0.005 & 0 & 0.0025 & 0.0255 & 3.64 & 4.28 & 0 & 1.90 & 12.47 & 17.66 & 3.96 & 0.02 & bal \\
\hline $\begin{array}{l}\text { KM4- } \\
\text { GB5 }\end{array}$ & 0.0081 & 0.0236 & 0.004 & 0.313 & 0.0023 & 0.0220 & 3.69 & 4.26 & 0 & 1.93 & 12.47 & 17.59 & 3.96 & 0.02 & bal \\
\hline $\begin{array}{l}\text { KM4- } \\
\text { GB6 }\end{array}$ & 0.0335 & 0.0231 & 0.003 & 0.320 & 0.0021 & 0.0235 & 3.71 & 4.30 & 0 & 1.93 & 12.34 & 17.63 & 3.98 & 0 & bal \\
\hline $\begin{array}{l}\text { KM4- } \\
\text { GB7 }\end{array}$ & 0.0106 & 0.0617 & 0.005 & 0.314 & 0.0016 & 0.0220 & 3.41 & 4.26 & 0 & 1.89 & 12.50 & 17.66 & 3.87 & 0.1 & bal \\
\hline $\begin{array}{l}\text { KM4- } \\
\text { GB8 }\end{array}$ & 0.0344 & 0.0633 & 0.003 & 0.319 & 0.0014 & 0.0235 & 3.81 & 4.38 & 0 & 1.97 & 12.30 & 17.56 & 4.01 & 0 & bal \\
\hline $\begin{array}{l}\text { KM4-CP } \\
\text { KM1 }\end{array}$ & 0.0190 & 0.0429 & 0.001 & 0.137 & 0.0046 & 0.0205 & 3.70 & 4.24 & 0 & 1.92 & 12.44 & 17.85 & 3.99 & 0.02 & bal \\
\hline $\begin{array}{l}\text { CH98 } \\
\text { (nominal) }\end{array}$ & 0.030 & 0.030 & & & & 0.030 & 4 & 4 & 4 & 0 & 12 & 18 & 4 & & bal \\
\hline
\end{tabular}

Table IV: Grain Boundary Element Matrix 2 Compositions (actual chemistries in w/o)

\begin{tabular}{|l|c|c|c|l|c|c|c|c|c|c|c|c|c|c|c|}
\hline Alloy & $\mathrm{B}$ & $\mathrm{C}$ & $\mathrm{S}$ & $\mathrm{Hf}$ & $\mathrm{Mg}$ & $\mathrm{Zr}$ & $\mathrm{Al}$ & $\mathrm{Ti}$ & $\mathrm{Ta}$ & $\mathrm{Nb}$ & $\mathrm{Cr}$ & $\mathrm{Co}$ & $\mathrm{Mo}$ & $\mathrm{W}$ & $\mathrm{Ni}$ \\
\hline GBE2-1 & 0.019 & 0.030 & $\mathrm{n} / \mathrm{a}$ & 0 & 0 & 0.040 & 3.90 & 4.02 & 0.83 & 0 & 12.52 & 19.20 & 3.79 & 0 & bal \\
\hline GBE2-2 & 0.020 & 0.054 & $\mathrm{n} / \mathrm{a}$ & 0 & 0.010 & 0.042 & 3.94 & 4.04 & 0.84 & 0 & 12.46 & 19.08 & 3.84 & 0 & bal \\
& & & & & & & & & & & & & & & \\
\hline GBE2-3 & 0.038 & 0.036 & $\mathrm{n} / \mathrm{a}$ & 0 & 0.013 & 0.038 & 3.86 & 3.74 & 0.84 & 0 & 12.46 & 19.25 & 3.86 & 0 & bal \\
\hline GBE2-4 & 0.040 & 0.055 & $\mathrm{n} / \mathrm{a}$ & 0 & 0 & 0.040 & 3.95 & 4.04 & 0.80 & 0 & 12.33 & 19.04 & 3.80 & 0 & bal \\
\hline GBE2-5 & 0.020 & 0.033 & $\mathrm{n} / \mathrm{a}$ & 0 & 0.004 & 0.040 & 4.07 & 4.02 & 2.30 & 0 & 12.33 & 19.18 & 3.80 & 0 & bal \\
\hline GBE2-6 & 0.020 & 0.054 & $\mathrm{n} / \mathrm{a}$ & 0 & 0 & 0.040 & 3.97 & 4.04 & 2.30 & 0 & 12.32 & 19.22 & 3.80 & 0 & bal \\
\hline GBE2-7 & 0.040 & 0.030 & $\mathrm{n} / \mathrm{a}$ & 0 & 0 & 0.040 & 3.99 & 4.04 & 2.30 & 0 & 12.35 & 18.95 & 3.82 & 0 & bal \\
\hline GBE2-8 & 0.040 & 0.044 & $\mathrm{n} / \mathrm{a}$ & 0 & 0.004 & 0.040 & 4.00 & 4.04 & 2.20 & 0 & 12.25 & 19.24 & 3.80 & 0 & bal \\
\hline GBE2-9 & 0.040 & 0.038 & $\mathrm{n} / \mathrm{a}$ & 0 & 0.004 & 0.040 & 4.07 & 4.03 & 1.60 & 0 & 12.35 & 18.91 & 3.83 & 0 & bal \\
\hline GBE2-10 & 0.030 & 0.030 & $\mathrm{n} / \mathrm{a}$ & 0 & 0 & 0.070 & 2.64 & 4.64 & 2.96 & 0 & 15.00 & 17.04 & 4.96 & 0 & bal \\
\hline
\end{tabular}




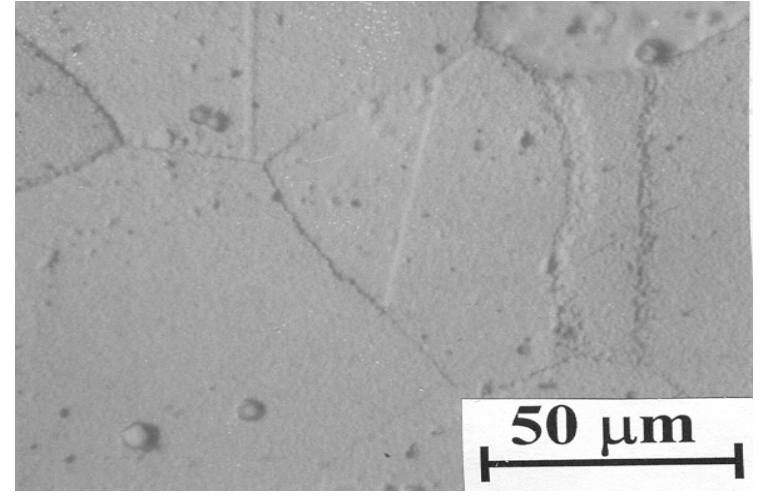

a) KM4-GB1 (low B / low C / low Hf / low Mg)

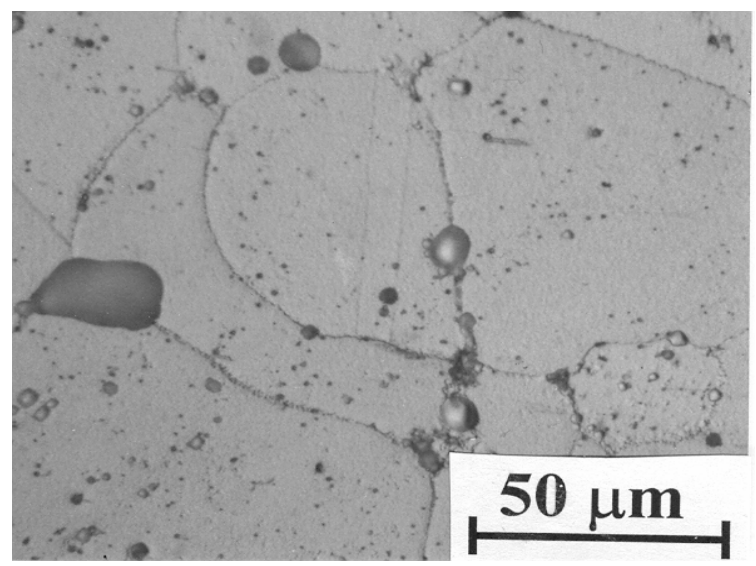

b) KM4-GB4 (high B / high C / low Hf / low Mg)

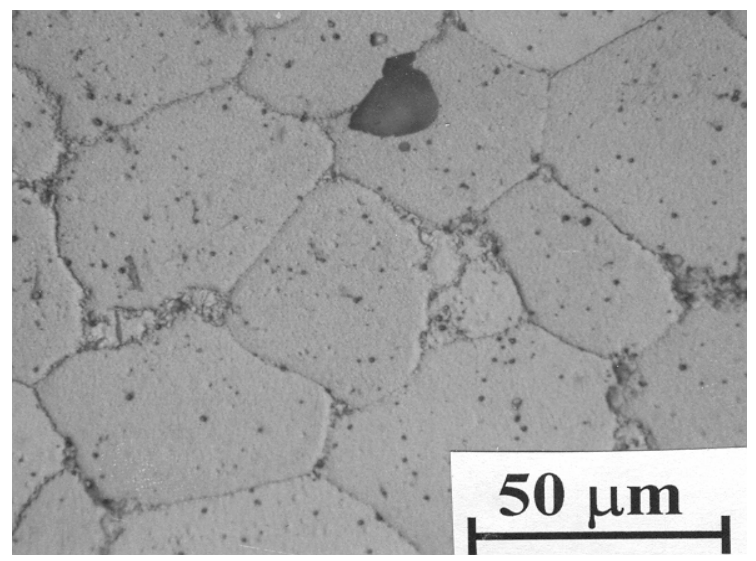

c) KM4-GB8 (high B / high C / high Hf / high Mg

Figure 2: Representative microstructures of supersolvus heat treated Matrix 1 material. Note increased amount of TIP and grain boundary decoration with increasing boron and carbon content. Some grain boundary eutectic phase was noted in the high Hf alloy (bottom photo).
The additional more detailed heat treat studies on Matrix 1 revealed further impacts of minor element additions. The TIP subjective scale rating and the TIP density change results showed a marked impact of boron (Table V).

Table V: TIP Results vs. Boron Level

\begin{tabular}{|l|c|c|c|}
\hline \multicolumn{1}{|c|}{ Alloy } & \multicolumn{1}{c|}{$\begin{array}{c}\text { Boron } \\
\text { Level }\end{array}$} & $\begin{array}{c}\text { Subjective TIP } \\
\text { Rating }\end{array}$ & $\begin{array}{c}\text { Density } \\
\text { Change (\%) }\end{array}$ \\
\hline $\begin{array}{l}\text { KM4- } \\
\text { GB1 }\end{array}$ & Lo / 0.01 & 1 & -0.096 \\
\hline $\begin{array}{l}\text { KM4- } \\
\text { GB2 }\end{array}$ & Hi / 0.04 & 3 & -0.34 \\
\hline $\begin{array}{l}\text { KM4- } \\
\text { GB3 }\end{array}$ & Lo / 0.01 & 1 & -0.073 \\
\hline $\begin{array}{l}\text { KM4- } \\
\text { GB4 }\end{array}$ & Hi / 0.04 & 3 & -1.44 \\
\hline $\begin{array}{l}\text { KM4- } \\
\text { GB5 }\end{array}$ & Lo / 0.01 & 2 & -0.089 \\
\hline $\begin{array}{l}\text { KM4- } \\
\text { GB6 }\end{array}$ & Hi / 0.04 & 3 & -0.43 \\
\hline $\begin{array}{l}\text { KM4- } \\
\text { GB7 }\end{array}$ & Lo / 0.01 & 1 & -0.10 \\
\hline $\begin{array}{l}\text { KM4- } \\
\text { GB8 }\end{array}$ & Hi / 0.04 & 3 & -0.44 \\
\hline $\begin{array}{l}\text { KM4- } \\
\text { GB-CP }\end{array}$ & 0.025 & 2 & -0.30 \\
\hline $\begin{array}{l}\text { KM4- } \\
\text { GB-Base }\end{array}$ & 0.03 & Not rated & Not rated \\
\hline KM4-Zr & 0.03 & 3 & -0.43 \\
\hline 456-GB8 & 0.05 & Not rated & Not rated \\
\hline CH98 & 0.03 & & \\
\hline
\end{tabular}

The average subjective rating for the low boron heats in Matrix 1 was 1.25 compared to an average of 3 for the high boron heats. For measured density change, the average density loss for the low boron heats was $0.09 \%$, while for the higher boron heats it was $0.66 \%$. This result matches prior work ${ }^{5}$. Similarly, the average of estimated incipient melting temperature for the low boron heats was $1241 \mathrm{C}$, compared to an average estimated value of $1191 \mathrm{C}$ for the high boron heats. Similar results were observed for Matrix 2.

The TIP subjective scale, TIP density change, and incipient melting were not statistically significant for any of the other elements in Matrix 1.

Heat treat studies to determine how GBE levels influenced grain boundary morphology for various thermal cycles were performed as well (Figure 3). Heat treatment cycles were done with a supersolvus solution temperature followed by single aging and duplex aging. The single aging was done at $760 \mathrm{C}$ for 8 hours; duplex cycles were $843 \mathrm{C} / 4$ hours plus $760 \mathrm{C}$ for 8 hours, and $899 \mathrm{C}$ for 2 hours plus $760 \mathrm{C}$ for 8 hours. The single aging cycle resulted in grain boundaries that were relatively free from continuous carbide films. Carbide phases were analyzed using scanning electron microscopy (SEM). As shown in Figure 4 , the structure contained both blocky $\mathrm{MC}$ carbides ( $\mathrm{Nb}$ rich) primarily within grain matrices, and $\mathrm{Cr}$ - and Mo- rich $\mathrm{M}_{23} \mathrm{C}_{6}$ carbides along grain boundaries. Small amounts of Hafnia were also present. 


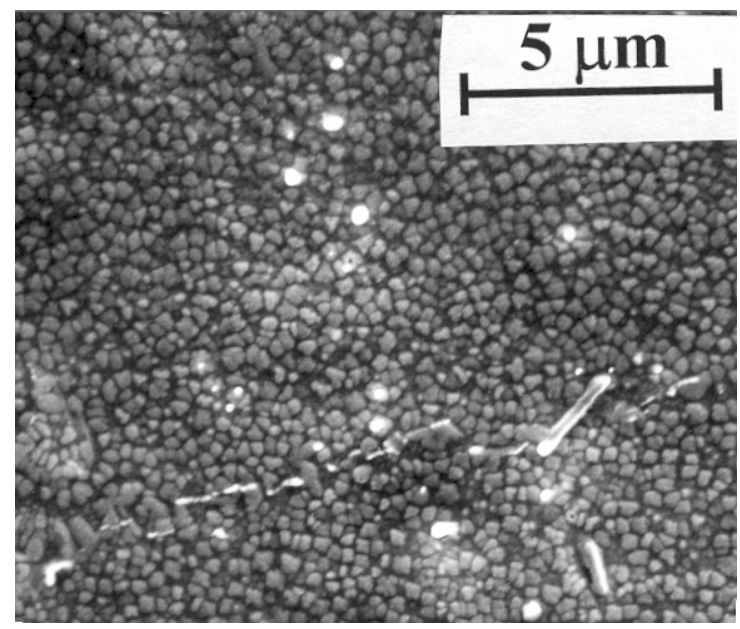

a) $760 \mathrm{C} / 8$ hour aging cycle.

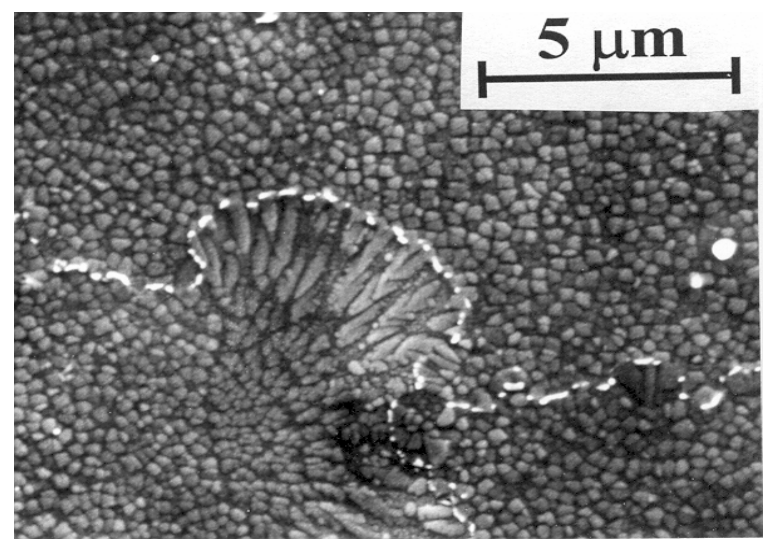

b) $843 \mathrm{C} / 4$ hour $+760 \mathrm{C} / 8$ hour aging cycle.

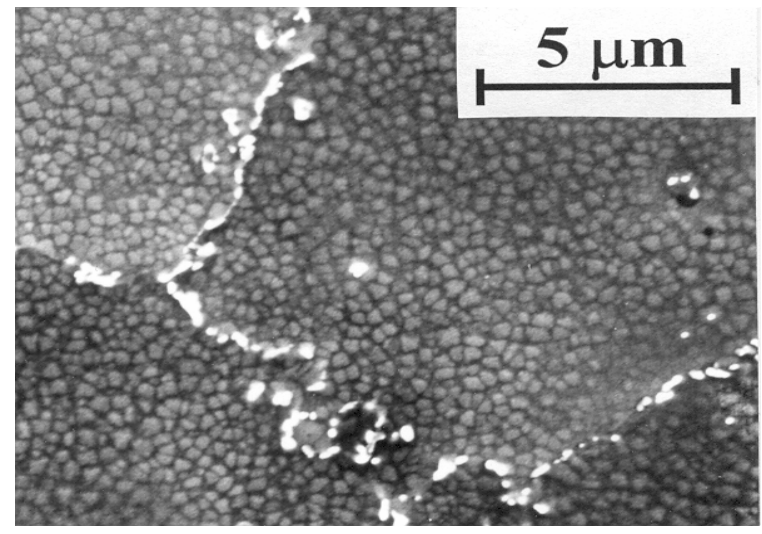

c) $899 \mathrm{C} / 4$ hour $+760 \mathrm{C} / 8$ hour aging cycle.

Figure 3: Microstructures vs. aging cycle. Carbide film formation was absent for the single age and increased with increasing age temperature for the duplex age cycles.

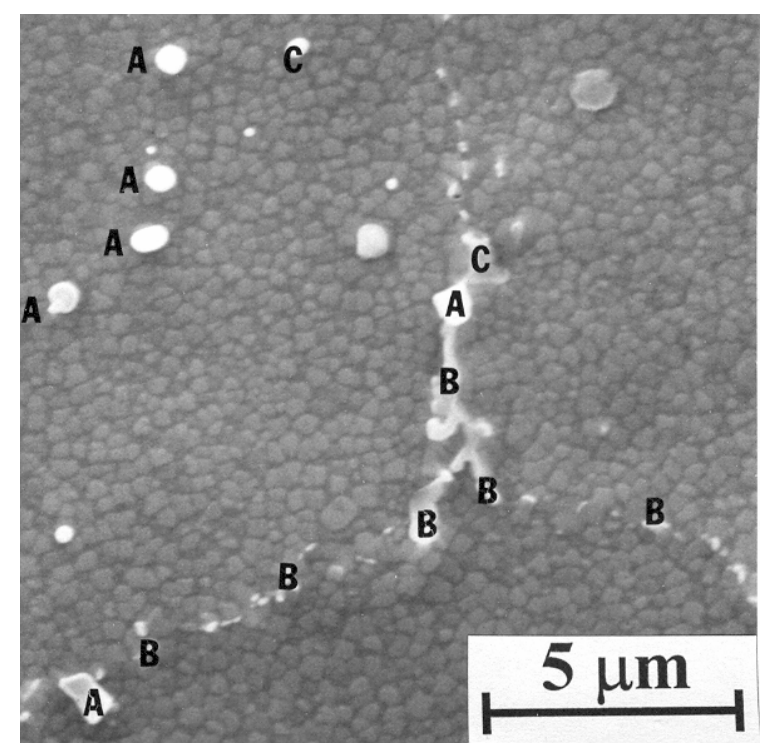

Figure 4: Typical carbides present in structure of alloy KM4GB8. Particle chemistries are: a) $\mathrm{Nb} / \mathrm{Ti} /$ trace $\mathrm{Hf}$; b) $\mathrm{Mo} / \mathrm{Cr}$ rich; c) Hf rich, with traces of $\mathrm{Zr}$.

Heat treatment studies to determine $\gamma^{\prime}$ solvus and grain growth behavior were done for both Matrix 1 and Matrix 2. For Matrix 1 , solvus temperatures were very consistent at $1177 \mathrm{C}$, except for $\mathrm{CH} 98$, with a solvus temperature of $1204 \mathrm{C}$ (note the $14 \mathrm{C}$ intervals influence the degree of fidelity of solvus temperature determination. For Matrix 2, solvus temperatures varied because of the Ta variation in the matrix. Solvus temperatures for the low $\mathrm{Ta}$ alloys were approximately $1177 \mathrm{C}$ and were approximately $1193 \mathrm{C}$ for the higher Ta alloys. Figure 5 shows the results for Matrix 2 alloys.

Overall mechanical property data are summarized in Tables VI and VII. Tests included tensile testing at $649 \mathrm{C}$, creep testing at $704 \mathrm{C}$, stress rupture testing at $649 \mathrm{C}$, and dwell Fatigue Crack Growth (FCG) testing at 704C. Duplicate tests were run where possible. Results were analyzed on both basis of general trends noted as well as a more rigorous analysis of statistical significance.

Tensile strength data for Matrix 1 are shown in Figure 6 . Tensile yield strengths $(0.2 \%$ offset $)$ at $649 \mathrm{C}$ ranged from 937 to $985 \mathrm{MPa}$. Ultimate Tensile Strength (UTS) values ranged from 1351 to $1386 \mathrm{MPa}$. Although not plotted, tensile ductility measurements were not impacted by composition. 
Table VI: Grain Boundary Element Matrix 1 Properties

\begin{tabular}{|c|c|c|c|c|c|c|c|c|c|c|}
\hline Alloy & B & $\mathrm{C}$ & Hf & $\mathrm{Mg}$ & $\begin{array}{l}649 \mathrm{C} \\
\mathrm{UTS}, \\
\mathrm{MPa} \\
(*)\end{array}$ & $\begin{array}{l}649 \mathrm{C} \\
0.2 \% \\
\mathrm{YS}, \mathrm{MPa} \\
(*)\end{array}$ & $\begin{array}{l}\text { Hrs to } \\
0.2 \% \\
\text { Creep } \\
649 \mathrm{C} \mathrm{/} \\
793 \mathrm{MPa} \\
(*)\end{array}$ & $\begin{array}{l}649 \mathrm{C} \\
\text { Rupture } \\
\text { Life } \\
\text { (hours) }\end{array}$ & $\begin{array}{l}\text { 649C } \\
\text { Rupture } \\
\text { Elongation } \\
(\%)\end{array}$ & $\begin{array}{l}704 \mathrm{C} \\
\mathrm{da} / \mathrm{dT} \text { at } 33 \\
\mathrm{MPa} \sqrt{\mathrm{m}}, 2 \\
\text { hour dwell }\end{array}$ \\
\hline KM4-GB1 & $\mathrm{Lo} / 0.01$ & $\mathrm{Lo} / 0.02$ & $\mathrm{Lo} / 0.0$ & $\mathrm{Lo} / 0.0$ & 1365.2 & 961.9 & 884.5 & 980 & 5.1 & $4.45 \mathrm{E}-7$ \\
\hline KM4-GB2 & $\mathrm{Hi} / 0.04$ & $\mathrm{Lo} / 0.02$ & $\mathrm{Lo} / 0.0$ & $\mathrm{Hi} / 0.005$ & 1358.3 & 958.4 & 669.5 & 2112 & 12.5 & $3.60 \mathrm{E}-7$ \\
\hline KM4-GB3 & $\mathrm{Lo} / 0.01$ & $\mathrm{Hi} / 0.06$ & $\mathrm{Lo} / 0.0$ & $\mathrm{Hi} / 0.005$ & 1375.6 & 958.4 & 542 & 590 & 3.25 & $1.30 \mathrm{E}-5$ \\
\hline KM4-GB4 & $\mathrm{Hi} / 0.04$ & $\mathrm{Hi} / 0.06$ & Lo/0.0 & Lo / 0.0 & 1372.1 & 955.0 & 163 & 1974 & 10.6 & $1.50 \mathrm{E}-5$ \\
\hline KM4-GB5 & $\mathrm{Lo} / 0.01$ & Lo / 0.02 & $\mathrm{Hi} / 0.3$ & $\mathrm{Hi} / 0.005$ & 1372.1 & 944.6 & 687 & 1023 & 4.15 & $1.15 \mathrm{E}-6$ \\
\hline KM4-GB6 & $\mathrm{Hi} / 0.04$ & Lo / 0.02 & $\mathrm{Hi} / 0.3$ & Lo / 0.0 & 1365.2 & 955.0 & 533.5 & 2058 & 16.85 & $8.05 \mathrm{E}-8$ \\
\hline KM4-GB7 & $\mathrm{Lo} / 0.01$ & $\mathrm{Hi} / 0.06$ & $\mathrm{Hi} / 0.3$ & Lo / 0.0 & 1351.4 & 937.7 & 604.5 & 742.5 & 3.45 & $1.80 \mathrm{E}-6$ \\
\hline KM4-GB8 & $\mathrm{Hi} / 0.04$ & $\mathrm{Hi} / 0.06$ & $\mathrm{Hi} / 0.3$ & $\mathrm{Hi} / 0.005$ & 1368.7 & 937.7 & 292 & 1946.5 & 17.25 & $6.05 \mathrm{E}-8$ \\
\hline $\begin{array}{l}\text { KM4-GB- } \\
\text { CP }\end{array}$ & 0.025 & 0.04 & 0.150 & 0.0025 & 1368.7 & 965.3 & 554.5 & 1544 & 12.7 & $2.24 \mathrm{E}-7$ \\
\hline $\begin{array}{l}\text { KM4-GB- } \\
\text { Base }\end{array}$ & 0.03 & 0.03 & 0.0 & 0.0 & 1368.7 & 958.4 & 554 & 2244.5 & 14.05 & $1.75 \mathrm{E}-7$ \\
\hline 456-GB8 & 0.05 & 0.07 & $\mathrm{Hi} / 0.3$ & $\mathrm{Hi} / 0.005$ & 1385.9 & 986.0 & 242 & 888 & 11.5 & $4.70 \mathrm{E}-7$ \\
\hline KM4-Zr & 0.03 & 0.3 & 0 & 0 & 1382.4 & 979.1 & No test & No test & No test & No test \\
\hline CH98 & 0.03 & 0.03 & 0 & 0 & 1372.1 & 961.9 & No test & No test & No test & No test \\
\hline Notes: & \multicolumn{10}{|c|}{ * average of two tests } \\
\hline
\end{tabular}

Table VII: Grain Boundary Element Matrix 2 Properties

\begin{tabular}{|c|c|c|c|c|c|c|c|c|c|c|}
\hline Alloy & B & $\mathrm{C}$ & $\mathrm{Ta}$ & $\mathrm{Mg}$ & $\begin{array}{l}649 \mathrm{C} \\
\mathrm{UTS}, \\
\mathrm{MPa} \\
(*)\end{array}$ & $\begin{array}{l}649 \mathrm{C} \\
0.2 \% \\
\mathrm{YS}, \mathrm{MPa} \\
(*)\end{array}$ & $\begin{array}{l}\text { Hrs to } \\
0.2 \% \\
\text { Creep } \\
649 \mathrm{C} \mathrm{/} \\
793 \mathrm{MPa} \\
(*)\end{array}$ & $\begin{array}{l}649 \mathrm{C} \\
\text { Rupture } \\
\text { Life } \\
\text { (hours) }\end{array}$ & $\begin{array}{l}\text { 649C } \\
\text { Rupture } \\
\text { Elongation } \\
(\%)\end{array}$ & $\begin{array}{l}704 \mathrm{C} \\
\mathrm{da} / \mathrm{dT} \text { at } 33 \\
\mathrm{Mpa} \sqrt{\mathrm{m}}, \quad 2 \\
\text { hour } \\
\text { dwell(*) }\end{array}$ \\
\hline GBE2-1 & Lo / 0.02 & Lo / 0.03 & Lo/0.8 & Lo / 0.0 & 1308.0 & 924.6 & 332.5 & 980 & 5.1 & $4.00 \mathrm{E}-7$ \\
\hline GBE2-2 & $\mathrm{Lo} / 0.02$ & $\mathrm{Hi} / 0.055$ & $\mathrm{Lo} / 0.8$ & $\mathrm{Hi} / .0075$ & 1306.6 & 950.1 & 259.5 & 2112 & 12.5 & $9.00 \mathrm{E}-8$ \\
\hline GBE2-3 & $\mathrm{Hi} / 0.04$ & Lo / 0.03 & $\mathrm{Lo} / 0.8$ & $\mathrm{Hi} / .0075$ & 1299.7 & 941.2 & 157 & 590 & 3.25 & $6.65 \mathrm{E}-7$ \\
\hline GBE2-4 & $\mathrm{Hi} / 0.04$ & $\mathrm{Hi} / 0.055$ & Lo/0.8 & Lo / 0.0 & 1304.5 & 955.0 & 163.5 & 1974 & 10.6 & $7.10 \mathrm{E}-7$ \\
\hline GBE2-5 & Lo / 0.02 & $\mathrm{Lo} / 0.03$ & $\mathrm{Hi} / 2.4$ & $\mathrm{Hi} / .0075$ & 1343.1 & 963.9 & 432 & 1023 & 4.15 & $4.00 \mathrm{E}-7$ \\
\hline GBE2-6 & Lo / 0.02 & $\mathrm{Hi} / 0.055$ & $\mathrm{Hi} / 2.4$ & Lo / 0.0 & 1352.8 & 962.5 & 326.7 & 2058 & 16.85 & $7.50 \mathrm{E}-8$ \\
\hline GBE2-7 & $\mathrm{Hi} / 0.04$ & Lo / 0.03 & $\mathrm{Hi} / 2.4$ & Lo / 0.0 & 1314.2 & 952.9 & 213.5 & 742.5 & 3.45 & $1.25 \mathrm{E}-7$ \\
\hline GBE2-8 & $\mathrm{Hi} / 0.04$ & $\mathrm{Hi} / 0.055$ & $\mathrm{Hi} / 2.4$ & $\mathrm{Hi} / .0075$ & 1348.0 & 971.5 & 331.5 & 1946.5 & 17.25 & $9.70 \mathrm{E}-8$ \\
\hline GBE2-9 & 0.030 & 0.043 & 1.60 & 0.0038 & 1293.5 & 930.8 & 150.4 & 1544 & 12.7 & $1.10 \mathrm{E}-7$ \\
\hline GBE2-10 & 0.03 & 0.03 & 0.0 & 0.0 & 1472.1 & 963.9 & 300.5 & 2244.5 & 14.05 & $6.50 \mathrm{E}-8$ \\
\hline Notes: & \multicolumn{10}{|c|}{$*$ average of two tests } \\
\hline
\end{tabular}




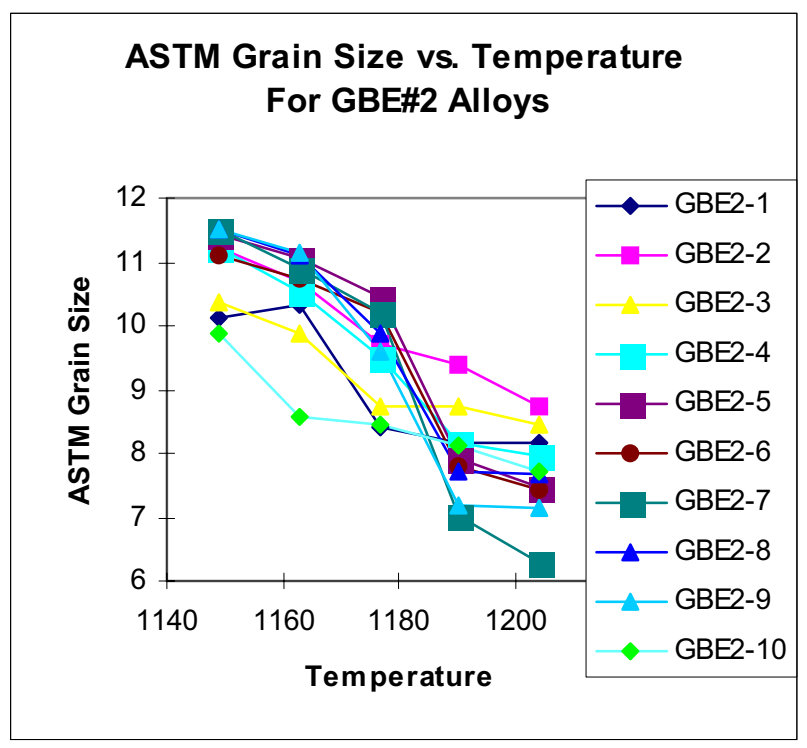

Figure 5: Grain Size vs. Temperature for Matrix 2

From a statistical standpoint, no element had clearly significant effects on either strength property, although $\mathrm{Hf}$ appeared to lower yield strength slightly and the Hf / Carbon interaction also correlated with lower yield strength (perhaps indicative that $\mathrm{Hf}$ carbide formation resulted in lower strength).

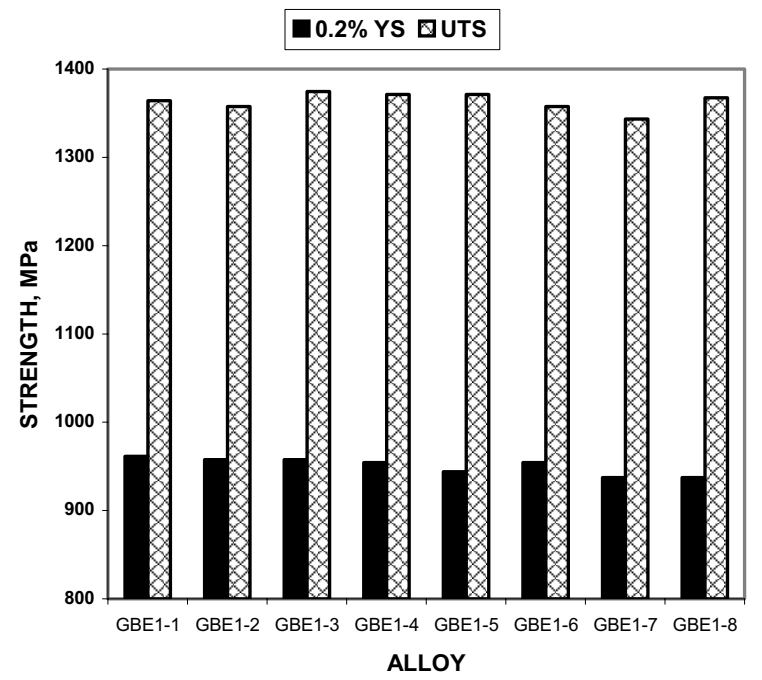

Figure 6: Tensile Strength for Matrix 1 Core Alloys

Creep lives are shown in Figure 7 for Matrix 1. Creep lives at 649C, $793 \mathrm{MPa}$ ranged from 163 to 884 hours. Statistical analysis showed that boron and carbon had statistically significant influence on this property, with increasing amounts of both predicted to lower creep life. The $\mathrm{B}+\mathrm{C}$ interaction is readily apparent in the pattern present in Figure 7 (low B, low C alloys GBE1-1 \& 1-5) and was significant statistically as well.

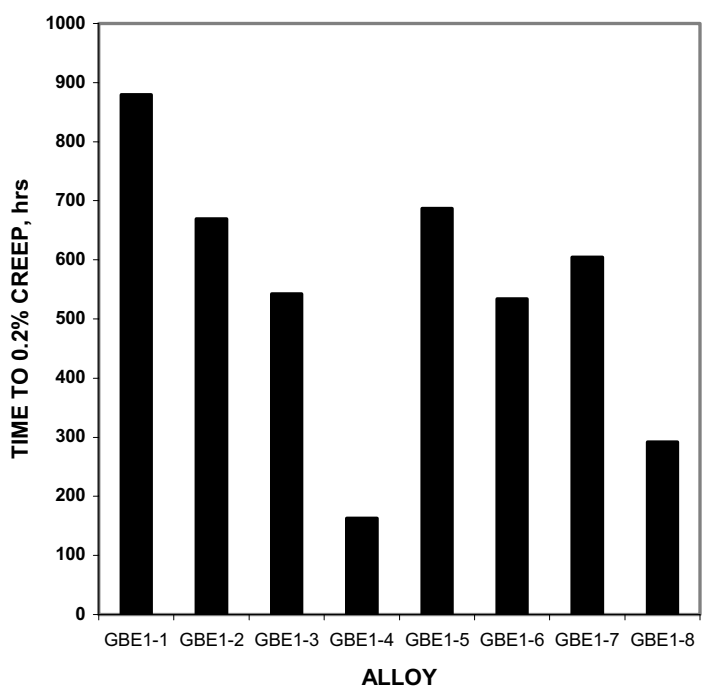

Figure 7: Creep Lives for Matrix 1 Core Alloys

Stress rupture lives for Matrix 1 are shown in Figure 8. Rupture lives at $649 \mathrm{C}, 896 \mathrm{MPa}$ ranged from 590 to 2244 hours, and rupture elongations ranged from 3.2 to $17 \%$. Both boron and magnesium contributed with statistical significance, with positive correlations for both, but the boron dominated the effect. Boron also significantly improved rupture elongation. The average elongation for the low boron heats was 3.9\% and the average elongation for the high boron heats was $14.3 \%$.

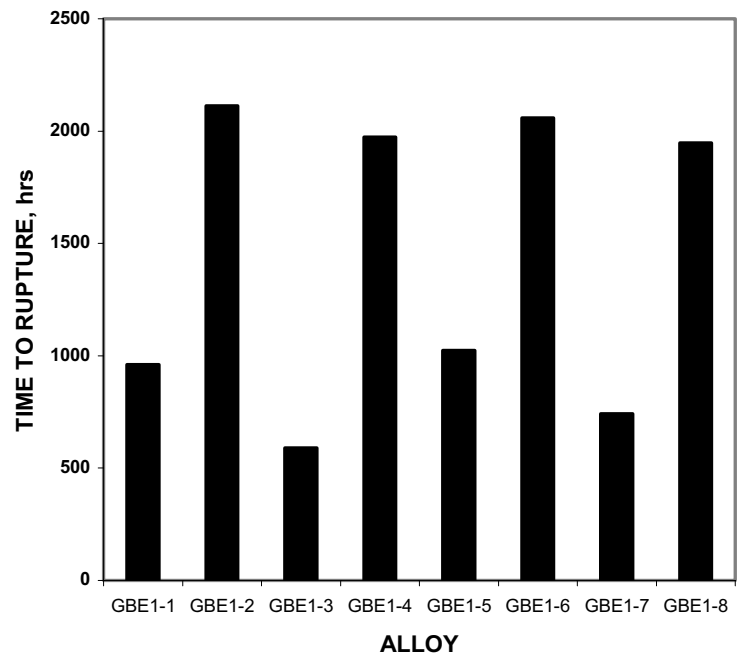

Figure 8: Rupture Lives for Matrix 1 Core Alloys 
Dwell crack growth data were run for all Matrix 1 alloys. When plotted on a crack growth rate per cycle vs. stress intensity basis, all alloys showed typical Paris Law behavior, but with some variation in thresholds and slope. Based on potential engine application conditions, the best crack growth comparison point was judged to be $33 \mathrm{Mpa} \sqrt{ } \mathrm{m}$. The data indicates that the lowest dwell FCG is for the combination of high B with high Hf. These data are shown in Figure 9.

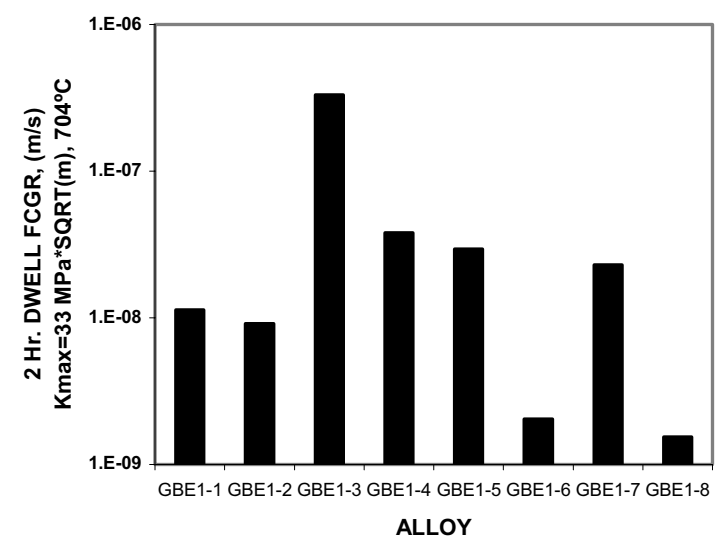

Figure 9: FCGR Comparisons for Matrix 1 Core Alloys

For Matrix 2, the trends for carbon and boron were similar to Matrix 1. Magnesium had a minor effect statistically but weakly debited $704 \mathrm{C}$ creep and slightly improved crack growth rate. The most marked impacts on properties were for Ta. Tantalum improved creep and strength, and had a positive impact on 704C hold time fatigue crack growth. By far the most marked impact was in crack growth. This is shown in Figure 10. In contrast to 704C / 2 hour dwell FCG, a few tests were done for 427C / 20 cpm crack growth, and there Ta had very little impact. Tantalum also improved creep as shown in Figure 11.

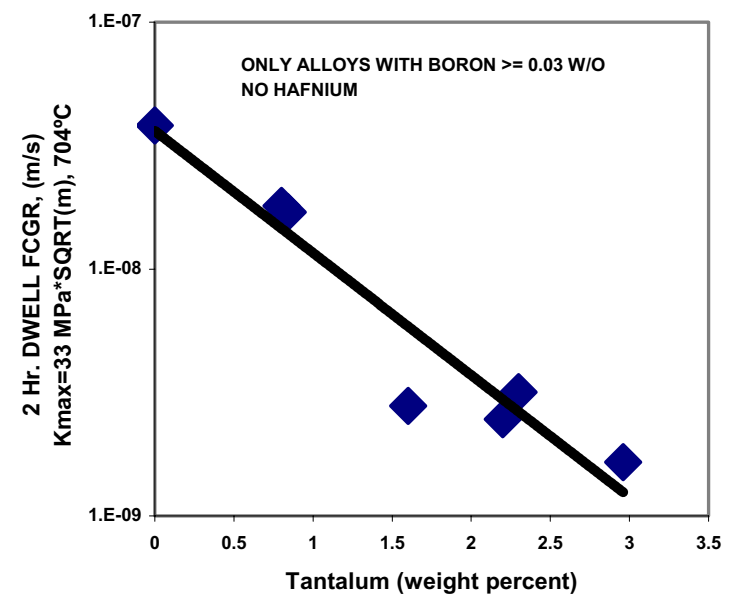

Figure 10: Influence of Ta on Hold Time Crack Growth (Matrix 2 Alloys)

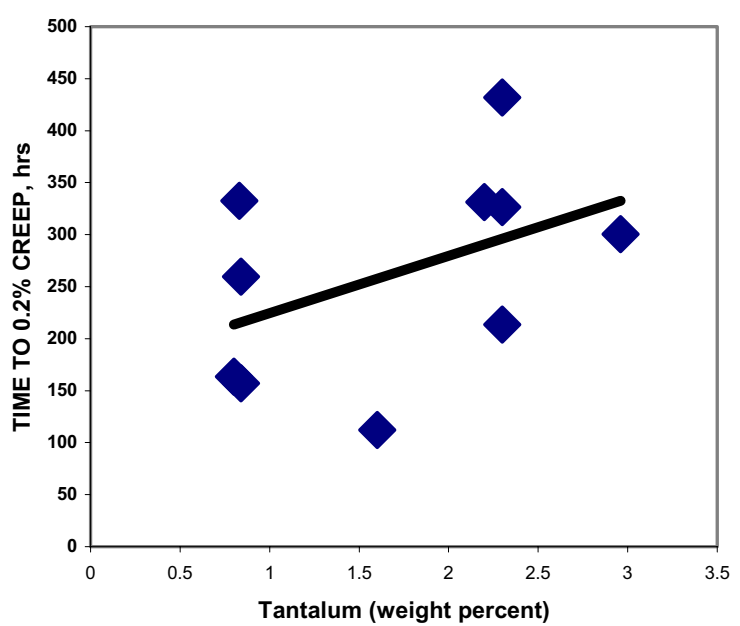

Figure 11: Influence of Ta on Creep (Matrix 2 Alloys)

\section{Discussion}

The dominance of $\mathrm{B}$ (and $\mathrm{Zr}$ ) on properties relative to $\mathrm{C}$ in both matrices has been reported for other $\mathrm{P} / \mathrm{M}$ alloy research (Reference 6). In this work $\mathrm{B}$ was seen to influence grain boundary strength and incipient melting at grain boundaries but not gamma-prime morphology. The authors suggested that the amount of B and other grain boundary elements be adjusted for grain size. For the study reported in this paper, the bulk of the work was done with supersolvus heat treated material in the ASTM 6-7 range of grain size. Limited work was done with both coarser and finer microstructures and suggested that minor element levels should in fact be tailored for grain size.

The relatively small influence of GBE variations on hold time crack growth was somewhat surprising. Many prior studies (for example, reference 7) have shown that removal of B and $\mathrm{Zr}$ can increase crack propagation rates. It is possible that the low level of $\mathrm{B}$ in matrix 1 and 2 was already above some minimum level to provide adequate dwell FCG rate resistance. The results suggest an intermediate B level should be used, driven by the need to compromise on the benefits of higher B on rupture and dwell FCG against the debits of higher B on creep rate and potentially on processing window as a result of boride melting during solution heat treatment. The interaction between $\mathrm{Hf}$ and $\mathrm{B}$ to reduce dwell FCG was also surprising. While hafnium additions were promising, some risk was assumed for possible impact of hafnium oxides on LCF initiation life and further work on hafnium was dropped.

The dominant impact on dwell FCG was observed for Ta. Tantalum has both a matrix impact and a somewhat indirect grain boundary modifier impact, as the formation of $\mathrm{TaC}$ influences the amount of carbon available for other carbides. The benefits of Ta on dwell FCG were as significant as Hf and did not carry the associated risks of Hf additions.

The overall trends of the experiment were used to guide grain boundary element levels chosen for the disk alloy development 
program. Subsequent alloy development efforts in the HSCT program were performed using these guidelines for grain boundary elements, while varying major elements, and led to compositions having desirable properties.

\section{Acknowledgements}

The authors gratefully acknowledge the financial support of NASA Glenn Research Center, under Government contract NAS3-26385. The sustained effort and effective team approach between NASA and both contractors for the HSCT program led to significant advances in understanding relationships between $\mathrm{P} / \mathrm{M}$ superalloy composition and processing and high temperature, long dwell properties. For NASA, Robert Draper was the focal point for the majority of the program; Draper was assisted by many colleagues, in particular Tim Gabb and Jack Telesman. The authors also acknowledge support of many colleagues including Bob VanStone, Joseph Heaney, Ron Tolbert and David Miller of GE; Herb Chin of P\&W; Jennifer Heine, formerly of P\&W and now at GE; and Mei Ling Henne, formerly of $\mathrm{P} \& \mathrm{~W}$ and now at PCC.

\section{References}

1. J. J. Schirra et. al., "Effect of Microstructure (and Heat Treatment) on the 649C Properties of Advanced PM Superalloy Disk Materials", elsewhere in these proceedings.

2. U. S. Patent 5,143,563, September 1, 1992.

3. Pratt \& Whitney, unpublished internal results.

4. U. S. Patent 5,662,749, June 7, 1995.

5. U. S. Patent 5,584,948, December 17, 1996.

6. T. J. Garosshen, T. D. Tillman, and G. P. McCarthy, "Effects of B, C, and $\mathrm{Zr}$ on the Structure and Properties of a P/M Nickel Base Superalloy", Met. Trans., 18A, 1987, pp. 69-77.

7. J. Gayda, T. P. Gabb, and R. V. Miner, "Fatigue Crack Propagation of Nickel Base Superalloys at 650C", NASA TM 87150. 
